\title{
Control biológico de Ctenarytaina eucalypti (Hem.: Psyllidae), plaga del eucalipto en Argentina
}

\author{
Biological control of Ctenarytaina eucalypti (Hem.: Psyllidae), pest of eucalyptus in Argentina
}

\author{
PATRICIO FIDALGO ${ }^{1}$, TANIA S. OLIVARES ${ }^{2}$, ANA MARIA DE HARO ${ }^{3}$ y JUSTO JOSE BARAÑAO ${ }^{4}$ \\ ${ }^{1}$ CRILAR-CONICET, Entre Ríos y Mendoza, 5301, Anillaco, La Rioja, Argentina. pfidalgo@crilar.com.ar \\ ${ }^{2}$ Universidad de Concepción, casilla 4040, Correo 3, Concepción, Chile. tolivare@udec.cl \\ ${ }^{3}$ Universidad Nacional de Luján, Depto. de Tecnología, Zoología Agrícola, CC221-6700 Luján, Buenos Aires, \\ Argentina. anadeharo@ciudad.com.ar \\ ${ }^{4}$ Universidad Nacional de Luján, Producción Vegetal IV, Dasonomía, CC221-6700 Luján, Buenos Aires, \\ Argentina.jbaranao@ciudad.com.ar
}

\section{SUMMARY}

To record the presence in Argentina of the parasitoid Psyllaephagus pilosus Noyes (Hym.: Encyrtidae) an specific natural enemy of a recent introduced pest of eucalyptus, Ctenarytaina eucalypti (Maskell) (Hemiptera: Psyllidae), is the objective of the present technical note. Although the home range of both species is Australia, they were accidentally introduced in our country. Some important characteristics of the pest and its biological control agent are mentioned, and the most relevant literature on the subject is provided.

Key words: eucalyptus, Ctenarytaina eucalypti, parasitoid, ecesis in biological control.

\section{RESUMEN}

El objetivo de la presente nota técnica es registrar la presencia en Argentina del parasitoide Psyllaephagus pilosus Noyes (Hym.: Encyrtidae), enemigo natural específico de una plaga de los eucaliptos recientemente introducida, Ctenarytaina eucalypti (Maskell) (Hemiptera: Psyllidae). Ambas especies son originarias de Australia e ingresaron accidentalmente en nuestro país. Se mencionan características importantes tanto de la plaga como de su agente de control y se brinda la bibliografía más importante relacionada con el tema.

Palabras clave: eucalipto, Ctenarytaina eucalypti, parasitoide, control biológico fortuito.

\section{INTRODUCCION}

Durante el "Primer Simposio Internacional del Eucalyptus globulus" realizado en Mar del Plata (Argentina) en el año 2000, se menciona por primera vez la presencia de una nueva plaga de los eucaliptos: Ctenarytaina eucalypti (Maskell) (Hemip.: Psyllidae) en la República Argentina, que ya estaría presente por lo menos desde 1998 $(1,2)$. Este psílido, conocido en el idioma inglés como "blue gum psyllid", es originario de Australia, desde donde en las últimas décadas del siglo pasado se dispersó a Nueva Zelandia, Sri Lanka, Sudáfrica y Europa (3). En 1991 fue detectado en California (4) y en 1999 en Chile (5). Productores afectados por la plaga en Argentina y que poseen plantaciones en Uruguay nos mencionaron que también está presente en este último país.

El ingreso accidental de la plaga a nuestro país se efectuó -afortunadamente- junto a su enemigo natural específico, el encírtido Psyllaephagus pilosus Noyes (Hymenoptera: Encyrtidae), por cuanto se trataría de un caso de "ecesis" o introducción fortuita junto a su huésped. 
BOSQUE 26(2): 91-93, 2005

Control biológico de Ctenarytaina eucalypti, plaga de eucalipto en Argentina

\section{MATERIAL Y METODOS}

Los ejemplares de $P$. pilosus y de $C$. eucalypti (ninfas y adultos) fueron colectados sobre plantas de E. globulus de 1 a 3 años en la región forestal de la provincia de Buenos Aires conocida como el "triángulo Balcarce-Mar del Plata-Necochea". Los ejemplares de C. eucalypti fueron identificados mediante comparación de los caracteres entregados por Burckhardt et al. (6) y se encuentran depositados como "voucher specimens" en las Colecciones Científicas de la Universidad de Concepción (UCCC) en Chile, mientras que los correspondientes a $P$. pilosus fueron determinados utilizando claves y descripciones $(7,8,9)$ y se encuentran depositados en el Museo de Historia Natural de Londres.

\section{RESULTADOS Y DISCUSION}

Ctenarytaina eucalypti (Maskell) pertenece al género Ctenarytaina Ferris \& Klyver, el que posee 25 especies conocidas en Australia y relacionadas a Eucalyptus y otras mirtáceas (10). El psílido o "piojo saltarín" se asemeja a una diminuta "chicharrita" (1,5 $\mathrm{mm}$ de largo) y tanto el adulto como las ninfas de ambos sexos se alimentan succionando savia de la planta, lo que hacen a través de un aparato bucal especializado con partes horadadoras y chupadoras, conocido como "rostrum".

La hembra adulta posee una coloración general púrpura oscura, casi negra. Vistas dorsalmente la cabeza y el tórax, de un púrpura oscuro, las alas amarillas, abdomen púrpura oscuro bandeado transversalmente con amarillo, antenas amarillas tornándose a negro en el ápice, ojos prominentes castaño oscuro, genitalia negra. Para más información y detalles ilustrados de la especie, recomendamos ver los trabajos 6, 11, 12.

Como ocurre con la mayoría de las plagas, $C$. eucalypti no posee importancia económica en su lugar de origen, Australia, donde se encuentra bajo un control biológico natural (13).

El ingreso de esta especie a nuestro país junto con un efectivo controlador natural ha evitado daños espectaculares y cuantiosos gastos en aplicaciones de insecticidas.

Luego de su establecimiento en California $P$. pilosus fue registrado en México (9) y recientemente fue importado en Chile para el control del psílido (11). Existe una recopilación (entre otras) de la información sobre el control biológico de esta plaga en el mundo hasta 1999 (14).

Psyllaephagus pilosus Noyes, 1988: 105-6. Los adultos de esta especie tienen un tamaño aproximado de $1 \mathrm{~mm}$, son de color oscuro (azul verdoso en la cabeza, casi negro en el tórax, castaño rojizo en el gáster y castaño oscuro en las patas), las alas son hialinas y el ovipositor (en la hembra) es poco o nada proyectante. La coloración del mesotórax varía según el sexo. En los machos el escudo verdoso contrasta con el escudete morado, mientras que en las hembras el escudo es ligeramente morado y el escudete suavemente bronceado; además, las setas de las antenas son comparativamente más largas en los machos que en las hembras. Para más detalles de la morfología e ilustraciones de las antenas, alas y genitalia del macho, ver los trabajos de Noyes (8) y Trjapitzin y Ruiz (9).

A $P$. pilosus originalmente se le asocia con $C$. eucalypti sobre E. globulus (8) y, a pesar que los materiales sobre los que basó la descripción el autor son de Nueva Zelandia, por la relación con Eucalyptus sugirió -acertadamente- un origen australiano para la especie. Se trata de un endoparasitoide primario y solitario de ninfas de psílidos. Las hembras de $P$. pilosus provocan heridas con el ovipositor en el cuerpo de ninfas jóvenes para que fluyan fluidos de los cuales se alimentan, es decir, causan daño al psílido durante el acto de oviposición (parasitación) y durante su propia alimentación o "host-feeding" $(3,15)$.

$P$. pilosus fue introducido con éxito en California, Gran Bretaña, Francia y Eire (14). En todos los casos se estableció rápidamente reduciendo significativamente la abundancia de las poblaciones de $C$. eucalypti; además, coloniza áreas adyacentes con facilidad. La lista de países donde fue introducido es extensa y se ha publicado una revisión de los programas llevados a cabo con este parasitoide y el éxito obtenido en cada caso (14).

\section{CONCLUSIONES}

El ingreso y establecimiento en Argentina de C. eucalypti no causó problemas en las plantaciones de eucaliptos, debido a que ingresó junto a su agente de control $P$. pilosus, un parasitoide 
muy relacionado a la plaga y a cuyas poblaciones mantiene a densidades por debajo del daño económico.

\section{AGRADECIMIENTOS}

Al Dr. John S. Noyes (Natural History Museum, London) por confirmar la identificación del agente de control (P. pilosus Noyes).

\section{BIBLIOGRAFIA}

(1) BLAZINA, A.M. Evolución alcanzada en las técnicas de vivero en los últimos cinco años. Primer Seminario Internacional de Eucalyptus globulus en la Argentina. Mar del Plata (Argentina) 6 y 7 de diciembre de 2000, p. $50-54$.

(2) DILLON, G. Aplicación de un conjunto de técnicas silviculturales intensivas en la producción de Eucalyptus globulus en la Argentina. Primer Seminario Internacional de Eucalyptus globulus en la Argentina. Mar del Plata (Argentina), 6 y 7 de diciembre de 2000, p. 32-49.

(3) DAHLSTEN, D.L., D.L. ROWNEY, W.A. COPPER, W.E. CHANEY, K.L. ROBB, S. TJOSVOLD, M. BIANCHI, P. LANE. Biological Control of the Blue Gum Psyllid in California. In: HODDLE, M.S. Proceedings of the First California Conference on Biological Control. June 10-11 University of California, Berkeley, 1998, p. $159-162$

(4) GILL, R. California Plant Pest and Disease Report. California Department of Food and Agriculture, 1991, vol. 10, p. 57

(5) SERVICIO AGRICOLA Y GANADERO, CHILE. El psílido de los eucaliptos Ctenarytaina eucalypti, Maskell (Hemiptera, Psyllidae). Bol. Inf. (Chile), 1999, 2 p.
(6) BURCKHARDT, D., D.L.Q. SANTANA, A.L. TERRA, F.M. de ANDRADE, S. PENTEADO, E. IEDE, C MOREY. Psyllids pest (Hemiptera: Psylloidea) in South American eucalypt plantations. Bulletin de la Société Entomologique Suisse, 1999, vol. 72, p. 1-10.

(7) NOYES, J.S. A review of the genera of Neotropical Encyrtidae (Hymenoptera: Chalcidoidea). Bull. Br. Mus. (N.H.) Entomol., 1980, Ser. 41, No 3, p. 107-253.

(8) NOYES, J.S. Encyrtidae (Insecta: Hymenoptera). Fauna of New Zealand, 1988, $\mathrm{N}^{\circ}$ 13, 188 p.

(9) TRJAPITZIN, V. A., E. RUIZ CANCINO. Encírtidos (Hymenoptera: Encyrtidae) de importancia agrícola en México. Serie Publicaciones Científicas CIDAFF-UAT, 2000, $162 \mathrm{p}$

(10) CARVER, M., G.F. GROSS, T.E. WOODWARD. Hemiptera (Bugs, leafhoppers, cicadas, aphids, scale insects, etc.). In: The Insects of Australia. A textbook for students and research workers. CSIRO Div. Ent., 1991, vol. II, p. 429-509.

(11) OLIVARES, T. S. Ctenarytaina eucalypti (Maskell, 1890): el psílido del eucalipto en Chile (Hemiptera: Sternorrhyncha: Psylloidea: Spondylaspininae). Gayana, 2000, vol. 64, No 2, p. 239-241.

(12) OLIVARES, T. S. Presencia de Ctenarytaina eucalypti (Maskell, 1890) en Chile (Hemiptera: Psyllidae). Res. XXII Congreso Nacional de Entomología. Sociedad Chilena de Entomología-Universidad Austral de Chile. Valdivia, Chile, 2000, p. 20.

(13) MORGAN, F.D. Handbook of the Flora and Fauna of South Australia. Government Printer, South Australia, 1984, p. 136.

(14) HODKINSON, I.D. Biocontrol of Eucalyptus psyllid Ctenarytaina eucalypti by the Australian parasitoid Psyllaephagus pilosus: a review of current programmes and their success. Biocontrol News and Information, 1999, 20 (4): 129-134.

(15) DAHLSTEN, D.L, D.L. ROWNEY, W.A. COPPER Landscapes: Integrating biological control of the eugenia psyllid into the day-to-day management of the landscape in Disneyland. In: HODDLE, M.S. Proceedings of the Second California Conference on Biological Control. July 11-12, The Historic Mission Inn, Riverside, California, 2000, p. 59-64. 